\title{
Silicon Drift Detectors in Electron Microscopy - An Over 20 Year History with a Bright Future
}

\author{
A.Liebel $^{1}$, A. Schöning ${ }^{1}$, A. Bechteler ${ }^{1}$, K. Hermenau ${ }^{1}$, K. Heinzinger ${ }^{2}$, L.Strüder ${ }^{3}$, A.Niculae ${ }^{1}$, \\ H.Soltau ${ }^{1}$ \\ ${ }^{1}$ PNDetector GmbH, Otto-Hahn-Ring 6, D-81739 München, Germany \\ ${ }^{2}$ PNSensor GmbH, Otto-Hahn-Ring 6, D-81739 München, Germany \\ ${ }^{3}$ University of Siegen, Department of Physics, Walter-Flex-Str. 3, 57068 Siegen, Germany
}

Initially developed as position-sensitive detectors for particle tracking [1], Silicon Drift Detectors (SDD) have become a standard tool in electron microscopy over the last two decades. The fact that these detectors are operated without liquid $\mathrm{N}_{2}$ cooling as well as the continuously improving performance have made them fast and efficient devices for Energy-Dispersive X-Ray Spectroscopy (EDS).

While the first employed SDD sensors had active areas of only $5 \mathrm{~mm}^{2}$ or $10 \mathrm{~mm}^{2}$, the detector size has been growing over the years, being driven by technological improvements and the market demand for increased signal intensity to achieve shorter measurement times. Nowadays, single channel SDDs with active areas up to $200 \mathrm{~mm}^{2}$ are available [2]. The use of oval shaped detectors (Fig. 1a) in Transmission Electron Microscopes (TEM) enables close proximity to the sample and increases the collection solid angle for the X-rays up to $1 \mathrm{sr}$. Annular four-channel SDDs (Fig. 1b) can be placed between pole piece and sample and expand the solid angle up to $1.8 \mathrm{sr}$. This allows for much shorter measurement time even with challenging samples. System-integrated EDS detectors in TEM led to a massive increase in solid angle and for the first time enabled elemental mappings in a reasonable time $[3,4]$.

The detection of low energy x-rays has become a more and more important task, especially in the field of low-voltage electron microscopy, where low energetic L- and M-lines are used for the elemental analysis. Modern scientific fields, such as energy storage materials demand for the detection of elements below Carbon. Windowless detectors with improved energy resolution and optimized radiation entrance window nowadays enable light element detection down to Lithium at only $54 \mathrm{eV}$ (Fig. 2b).

The continuous improvement of Silicon Drift Detectors with internal JFET [5] as well as new preamplifier concepts for SDDs with external transistor [6] have led to detectors with better energy resolution and excellent high count rate performance. The energy resolution could meanwhile be enhanced down to very good values of 121 eV FWHM @ Mn-Ka (Fig. 2a). Newest detectors can achieve energy resolution values that are sufficiently good for many applications even at chip temperatures close to room temperature [5]. This opens the door to compact and innovative new detector designs where only a minimum cooling is required.

This contribution will give a brief overview on the history and developments in the field of Silicon Drift Detectors in microanalysis over the past 20 years. Within this, we will concentrate on how applications in electron microscopy and consequently challenges for the detectors evolved over the years and how the devices had to be adapted to this. Developments, such as larger active areas, advanced chip geometries, new read-out concepts for improved high count rate performance as well as systemintegrated SDDs in Transmission Electron Microscopy will be considered. 
References:

[1] L Strüder et al, Microscopy \& Microanalysis, volume 4, (1999), pp. 622-631

[2] A Niculae et al, Proceedings of Microscopy \& Microanalysis, volume 22, S3 (2016), pp. $42-43$

[3] P Schlossmacher et al, Microscopy Today, vol. 18(4), (2010), pp. 14-20

[4] HS von Harrach et al, Journal of Physics Conference Series, volume 241(1), (2010)

[5] A Niculae et al, Proceedings of Microscopy \& Microanalysis, volume 22, S3 (2016), pp. 40-41

[6] L. Bombelli et al, IEEE Nuclear Science Symposium Conference (NSS/MIC), ISBN 978-1-4244-

9105-6 (2010)

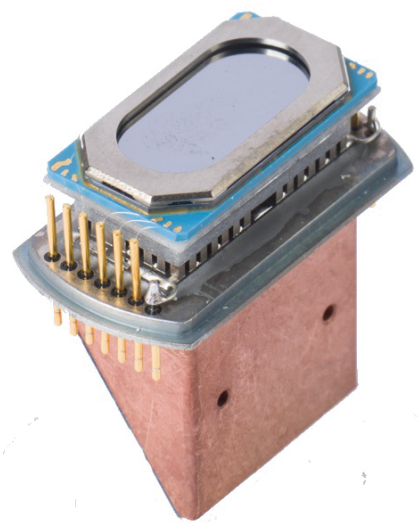

a)

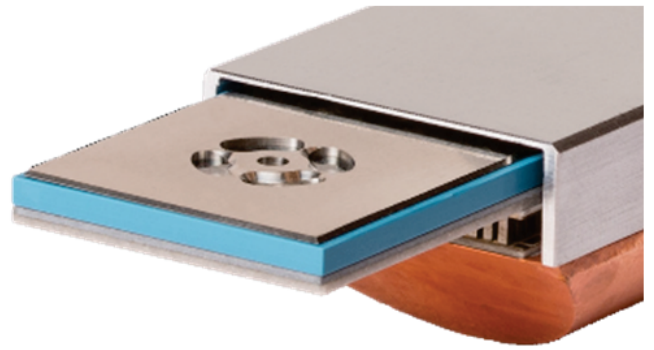

b)

Figure 1. Silicon Drift Detectors for high solid angle applications. a) The $100 \mathrm{~mm}^{2}$ SDD Racetrack with its oval shaped active area can achieve solid angles up to $1 \mathrm{sr}$. b) The annular 4-channel SDD Rococo3 can be positioned very close to the sample which leads to extreme solid angles up to $1.8 \mathrm{sr}$.

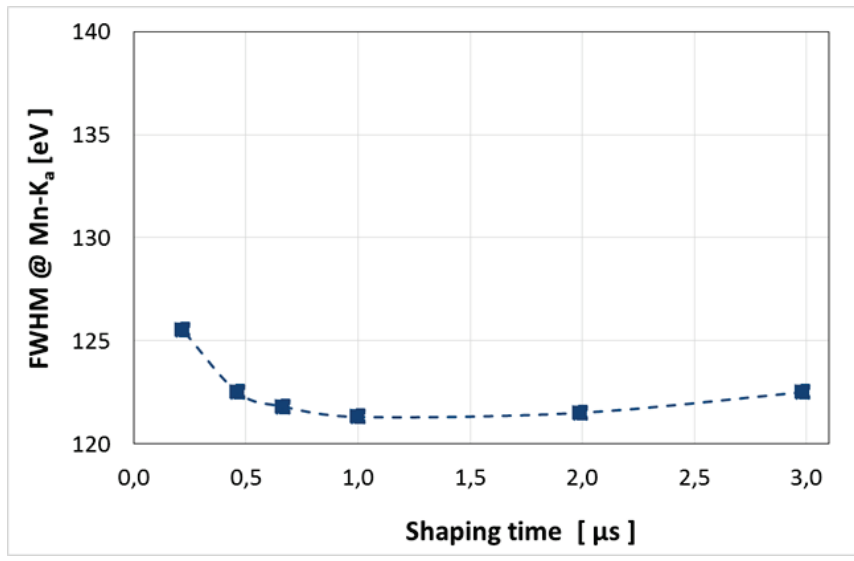

a)

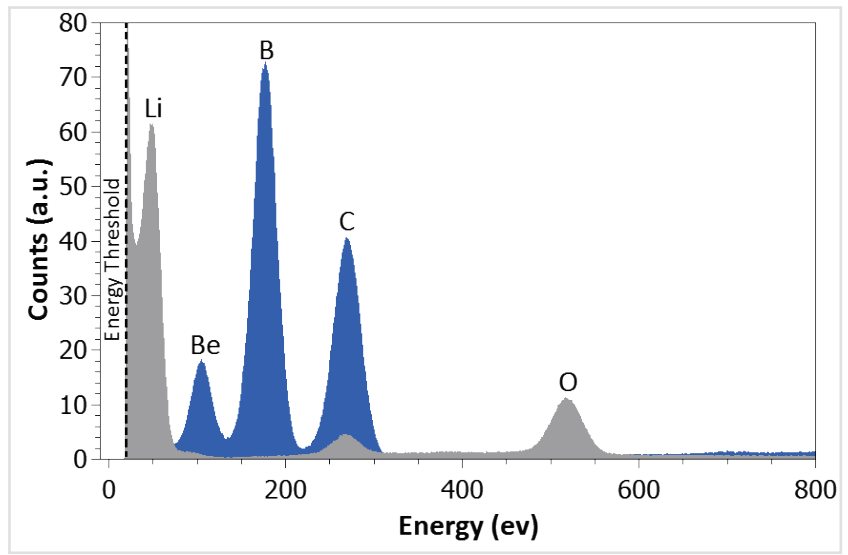

b)

Figure 2. Results from a $10 \mathrm{~mm}^{2}$ Silicon Drift Detector of the newest generation $\mathrm{SDD}^{\mathrm{GL}}$ with a) excellent energy resolution of $121.5 \mathrm{eV} @ \mathrm{Mn}-\mathrm{K} \alpha, 1 \mu \mathrm{s}$ shaping time and $126 \mathrm{eV} @ \mathrm{Mn}-\mathrm{K} \alpha, 0.22 \mu \mathrm{s}$ shaping time and b) two superposed light element spectra showing all light elements from Lithium to Carbon well separated from each other. 